\title{
Integral holomorphic functions
}

\author{
by \\ Verónica Dimant (Buenos Aires), Pablo Galindo (Valencia), \\ Manuel Maestre (Valencia) and Ignacio Zalduendo (Buenos Aires)
}

\begin{abstract}
We define the class of integral holomorphic functions over Banach spaces; these are functions admitting an integral representation akin to the Cauchy integral formula, and are related to integral polynomials. After studying various properties of these functions, Banach and Fréchet spaces of integral holomorphic functions are defined, and several aspects investigated: duality, Taylor series approximation, biduality and reflexivity.
\end{abstract}

Introduction. In this paper we define and study a class of holomorphic functions over infinite-dimensional Banach spaces admitting integral representation. Our purpose, and the motivation for our definition, are two-fold: we wish to obtain an integral representation formula akin to the Cauchy integral formula valid for some holomorphic functions over a Banach space, and we also wish to obtain a duality theorem generalizing those of Sebastião e Silva and Köthe $[\mathrm{S}],[\mathrm{K}]$.

In the 1950's Sebastião e Silva and Köthe studied the dual of $\mathcal{H}(U)$, where $U$ is an open subset of the Riemann sphere. They found that $\mathcal{H}(U)^{\prime}$ can be represented as the algebra of germs of analytic functions on $K=U^{\mathrm{c}}$ via the duality $\mathcal{H}(U) \times \mathcal{O}(K) \rightarrow \mathbb{C}$ given by

$$
\langle f, h\rangle=\frac{1}{2 \pi i} \int_{\Gamma} h(\lambda) f(\lambda) d \lambda
$$

where $\Gamma$ is a curve around $K$ contained in the domain of $h$. Thus one obtains an isomorphism $B: \mathcal{H}(U)^{\prime} \rightarrow \mathcal{O}(K)$ given by $B(T)(\omega)=T\left(f_{\omega}\right)$, where $f_{\omega}(x)=1 /(x-\omega)$. As it stands, this of course cannot be done on an arbitrary Banach space. However, we may look at the duality above after a change of variables (and reversal of circulation) as $\mathcal{H}(U) \times \mathcal{O}\left(K^{-1}\right) \rightarrow \mathbb{C}$

2000 Mathematics Subject Classification: Primary 46G20, 46E50; Secondary 46E15, 46E10, $46 \mathrm{~B} 10$.

The research of Galindo was supported in part by DGESIC Project no. PB96-0758.

The research of Maestre was supported in part by MCYT and FEDER Project BFM2002-01423. 
with

$$
\langle f, g\rangle=\frac{1}{2 \pi i} \int_{\Gamma^{-1}} g(\omega) \frac{f(1 / \omega)}{\omega} d \omega=\int_{\Gamma^{-1}} g(\omega) f(1 / \omega) \frac{d \omega}{2 \pi i \omega} .
$$

Once this is done, one has the isomorphism $B: \mathcal{H}(U)^{\prime} \rightarrow \mathcal{O}\left(K^{-1}\right)$, where $B(T)(\omega)=T\left(f_{\omega}\right)$, with $f_{\omega}(x)=1 /(1-\omega x)$. This can be generalized to a Banach space $E$ if we are willing to accept $\omega \in E^{\prime}$.

We also want to obtain a representation similar to the Cauchy integral formula for some analytic functions over a Banach space. The usual generalization of the Cauchy formula to infinite dimensions is very useful, but is really just the one-dimensional formula in each direction: for $x \in E$ and $|z|<r$

$$
h(z x)=\frac{1}{2 \pi i} \int_{|\lambda|=r} \frac{h(\lambda x)}{\lambda-z} d \lambda .
$$

But if we return to the one-dimensional formula and change variables we have, for $f$ analytic near the unit $\operatorname{disc} \Delta$, and $x \in \Delta^{\circ}$,

$$
f(x)=\frac{1}{2 \pi i} \int_{T} \frac{1}{1-\omega x} \frac{f(1 / \omega)}{\omega} d \omega=\int_{T} \frac{1}{1-\omega x} f(1 / \omega) \frac{d \omega}{2 \pi i \omega} .
$$

We want to generalize this to the infinite-dimensional setting by replacing the Cauchy kernel $1 /(\lambda-x)$ with $1 /(1-\gamma(x))$, where $x \in E$ has norm less than one, and $\gamma \in E^{\prime}$ is in the unit ball.

In Section 1 we define and study integral holomorphic functions, as well as the space of all such functions on the open unit ball of $E$, which we denote by $\mathcal{H}_{\mathrm{I}}\left(B_{E}^{\circ}\right)$. We also present a duality result referred to above. Since in this space the Taylor series expansion does not have good convergence properties, as we shall see, we introduce related spaces, $\mathcal{H}_{\mathrm{bI}}\left(B_{E}^{\circ}\right)$ and $\mathcal{H}_{\mathrm{bI}}(E)$, in Section 2, in which the Taylor series is better behaved. Finally, in Section 3, we study the biduality and reflexivity of these spaces.

The authors have received valuable comments and remarks regarding the content of this paper from several colleagues, including Oscar Blasco, Daniel Carando, Seán Dineen and Domingo García. It is a pleasure to acknowledge their help.

1. Integral holomorphic functions and the space $\mathcal{H}_{\mathrm{I}}\left(B_{E}^{\circ}\right)$. Integral polynomials were introduced by Dineen in [D1] and [D2]. An $n$-homogeneous polynomial $P: E \rightarrow \mathbb{C}$ over $E$ is said to be integral if it can be represented as

$$
P(x)=\int_{B_{E^{\prime}}} \gamma(x)^{n} d \mu(\gamma)
$$

for all $x \in E$, where $\mu$ is a regular Borel measure on $\left(B_{E^{\prime}}, w^{*}\right)$. Of course many measures may represent the same polynomial $P$. The integral norm 
of $P$ is defined as

$$
\|P\|_{\mathrm{I}}=\inf \{|\mu|: \mu \text { represents } P\} .
$$

With this norm, the space of all $n$-homogeneous integral polynomials over $E$, $P_{\mathrm{I}}\left({ }^{n} E\right)$, is a Banach space. It is in fact the dual of the complete symmetric $n$ fold $\varepsilon$-tensor product $\widehat{\bigotimes}_{n, s, \varepsilon} E$ (this result was first proved by Dineen in [D2] when $E$ is a dual space), or equivalently, the dual of the subspace of $C\left(B_{E^{\prime}}\right)$ spanned by the $w^{*}$-continuous functions $\gamma \mapsto \gamma(x)^{n}$ for $x \in E$. All nuclear polynomials are integral, and if $E^{\prime}$ has the Radon-Nikodym property, the spaces of nuclear and integral polynomials coincide isomorphically $[\mathrm{A}]$ and isometrically $[\mathrm{CD}],[\mathrm{BR}]$. For more on integral polynomials see [D3, Section $2.3]$.

Definition 1. We will say that a function $f: B_{E}^{\circ} \rightarrow \mathbb{C}$ is integral if there is a regular Borel measure $\mu$ on $\left(B_{E^{\prime}}, w^{*}\right)$ such that for every $x$,

$$
f(x)=\int_{B_{E^{\prime}}} \frac{1}{1-\gamma(x)} d \mu(\gamma) .
$$

On the space of all such functions, which we will denote by $\mathcal{H}_{I}\left(B_{E}^{\circ}\right)$, we define the norm

$$
\|f\|=\inf \{|\mu|: \mu \text { represents } f\} .
$$

The following properties of integral functions are very elementary and for this reason we do not prove them here.

Proposition 2. Let $f$ and $\mathcal{H}_{\mathrm{I}}\left(B_{E}^{\circ}\right)$ be as above. Then

(i) $f$ is holomorphic on $B_{E}^{\circ}$. In fact, for each $a \in B_{E}^{\circ}$ the $k$-homogeneous polynomial in the Taylor series expansion of $f$ about $a$ is

$$
P(x)=\int_{B_{E^{\prime}}} \frac{\gamma(x)^{k}}{(1-\gamma(a))^{k+1}} d \mu(\gamma) .
$$

(ii) $\left(\mathcal{H}_{\mathrm{I}}\left(B_{E}^{\circ}\right),\|\cdot\|_{\mathrm{I}}\right)$ is a Banach space.

Note that (i) above implies that the Taylor series expansion of an integral function involves only integral polynomials, and one has the Cauchy-type inequalities

$$
\|P\|_{\mathrm{I}} \leq \frac{\|f\|_{\mathrm{I}}}{(1-\|a\|)^{k+1}} .
$$

For $a=0$, if $f=\sum_{k} P_{k}$ is the Taylor series expansion of $f$ around 0 , one has $\left\|P_{k}\right\| \leq\left\|P_{k}\right\|_{\mathrm{I}} \leq\|f\|_{\mathrm{I}}$, so the radius of convergence of the series is

$$
r_{\mathrm{c}}=\frac{1}{\limsup \left\|P_{k}\right\|^{1 / k}} \geq 1 \text {. }
$$

Thus integral functions are holomorphic of bounded type (though not necessarily bounded) on the open unit ball of $E$. In fact, the mapping $\mathcal{H}_{\mathrm{I}}\left(B_{E}^{\circ}\right) \rightarrow$ 
$\mathcal{H}_{\mathrm{b}}\left(B_{E}^{\circ}\right)$ is continuous, though not closed. Note that though the sequence $\left(\left\|P_{k}\right\|_{\mathrm{I}}\right)$ is bounded, it need not belong to any $\ell^{p}$ space: indeed, if $\varphi \in E^{\prime}$ has norm one, then $f(x)=\sum_{k} \varphi(x)^{k}$ is integral (with representing measure $\left.\delta_{\varphi}\right)$ but $\left\|P_{k}\right\|_{\mathrm{I}}=1$ for all $k$.

Integral polynomials are integral holomorphic functions, and their norms as integral functions coincide with their norms as integral polynomials. In fact, the following proposition holds.

Proposition 3. If $f=\sum_{k} P_{k}$, where $P_{k}$ are $k$-homogeneous integral polynomials and the sequence $\left(\left\|P_{k}\right\|_{\mathrm{I}}\right)_{k}$ is summable, then $f$ is integral, with integral norm not exceeding the $\ell^{1}$-norm of $\left(\left\|P_{k}\right\|_{\mathrm{I}}\right)_{k}$.

Proof. Let $\varepsilon>0$, and for each $k=0,1, \ldots$ take $\mu_{k}$ to be a measure representing $P_{k}$ as a $k$-homogeneous polynomial:

$$
P_{k}(x)=\int_{B_{E^{\prime}}} \gamma(x)^{k} d \mu_{k}(\gamma)
$$

and with total variation $\left\|\mu_{k}\right\|$ bounded above by $\left\|P_{k}\right\|_{\mathrm{I}}+\varepsilon / 2^{k}$. Let $M_{k}$ be the closed subspace of $C\left(B_{E^{\prime}}, w^{*}\right)$ spanned by $\left\{\widehat{x}^{k}: x \in E\right\}$, and denote by $M$ the direct sum of these over $k$. Define $\mu \in M^{\prime}$ by $\mu(Q)=\sum_{k=0}^{m} \mu_{k}\left(Q_{k}\right)$ if $Q=\sum_{k=0}^{m} Q_{k}$ with $Q_{k} \in M_{k}$. We check the continuity of $\mu$ :

$$
|\mu(Q)|=\left|\sum_{k=0}^{m} \mu_{k}\left(Q_{k}\right)\right| \leq \sum_{k=0}^{m}\left\|\mu_{k}\right\|\left\|Q_{k}\right\|,
$$

which by the Cauchy inequalities is no larger than

$$
\|Q\| \sum_{k=0}^{m}\left\|\mu_{k}\right\| \leq\|Q\|\left(\sum_{k=0}^{m}\left\|P_{k}\right\|_{\mathrm{I}}+\frac{\varepsilon}{2^{k}}\right) \leq\|Q\|\left(\sum_{k=0}^{\infty}\left\|P_{k}\right\|_{\mathrm{I}}+\varepsilon\right) .
$$

Thus $\mu$ extends to the closure of $M$, and by Hahn-Banach to all of $C\left(B_{E^{\prime}}, w^{*}\right)$ conserving its norm, thus $\|\mu\| \leq \sum_{k=0}^{\infty}\left\|P_{k}\right\|_{\mathrm{I}}+\varepsilon$ as one wants. We have $P_{k}(x)=\int \gamma(x)^{k} d \mu(\gamma)$, and then

$$
f(x)=\sum_{k=0}^{\infty} \int_{B_{E^{\prime}}} \gamma(x)^{k} d \mu(\gamma)=\int_{B_{E^{\prime}}} \frac{1}{1-\gamma(x)} d \mu(\gamma) .
$$

ExAmple 4. Let $E=\ell^{2}$ and $P_{k}(x)=a_{k} x_{k}^{k}$, with $\left(a_{k}\right)$ a bounded non-summable sequence of positive numbers. We have $\left\|P_{k}\right\|_{\mathrm{I}}=a_{k}$. Since $a_{k} e_{k}^{\prime} \rightarrow 0$ weakly, $f=\sum_{k} P_{k}$ is holomorphic on $\ell^{2}$. If $f$ were integral, there would be a measure $\mu$ such that for all $x$,

$$
f(x)=\int_{B_{\ell^{2}}} \frac{1}{1-\gamma(x)} d \mu(\gamma)
$$


and thus

$$
P_{k}(x)=\int_{B_{\ell^{2}}} \gamma(x)^{k} d \mu(\gamma)
$$

Now, for each $N \geq 2$, let $h_{N}=\sum_{k=2}^{N} e_{k}^{k}$. These are weakly continuous functions on the unit ball and each $h_{N}$ has norm one: $h_{N}\left(e_{2}^{\prime}\right)=1$, and

$$
\left\|h_{N}\right\|=\sup _{\gamma \in B_{\ell^{2}}}\left|\sum_{k=2}^{N} \gamma_{k}^{k}\right| \leq \sup _{\gamma \in B_{\ell^{2}}} \sum_{k=2}^{N}\left|\gamma_{k}\right|^{k} \leq \sup _{\gamma \in B_{\ell^{2}}} \sum_{k=2}^{N}\left|\gamma_{k}\right|^{2}=1 .
$$

However,

$$
\begin{aligned}
\left|\mu\left(h_{N}\right)\right| & =\left|\int_{B_{\ell^{2}}} h_{N}(\gamma) d \mu(\gamma)\right|=\left|\sum_{k=2}^{N} \int_{B_{\ell^{2}}} e_{k}^{k}(\gamma) d \mu(\gamma)\right| \\
& =\left|\sum_{k=2}^{N} P_{k}\left(e_{k}\right)\right|=\sum_{k=2}^{N} a_{k},
\end{aligned}
$$

which tends to infinity with $N$, so $\mu$ is not bounded, absurd; hence $f$ is not integral.

Proposition 3 is sharp in the sense that one can have $\left(\left\|P_{k}\right\|_{\mathrm{I}}\right)_{k} \in \ell^{p}$ for all $p>1$, and $f$ not integral. It suffices to take in the above example $\left(a_{k}\right)$ a non-summable sequence of positive numbers which is $p$-summable for all $p>1$.

We now present a duality result analogous to those of Sebastião e Silva and Köthe referred to in the Introduction.

THEOREM 5. $\mathcal{H}_{\mathrm{I}}\left(B_{E}^{\circ}\right)$ is a dual space. In fact, if $E$ has the approximation property, then $\mathcal{H}_{\mathrm{I}}\left(B_{E}^{\circ}\right)=A\left(B_{E^{\prime}}\right)$ ' (i.e., the dual of the "ball algebra" of analytic functions in $B_{E^{\prime}}^{\circ}$ which are $w^{*}$-continuous in $B_{E^{\prime}}$.) This duality has an integral representation $\mathcal{H}_{\mathrm{I}}\left(B_{E}^{\circ}\right) \times A\left(B_{E^{\prime}}\right) \rightarrow \mathbb{C}$ given by

$$
\langle f, g\rangle=\int_{B_{E^{\prime}}} g(\gamma) d \mu(\gamma)
$$

where $\mu$ represents $f$.

Proof. Recall that $P_{\mathrm{I}}\left({ }^{k} E\right)$ is the dual of the symmetric $\varepsilon$-tensor product $\bigotimes_{\varepsilon, k, s} E$ (cf. [CZ]), which can be identified with the closed subspace of $C\left(B_{E^{\prime}}, w^{*}\right)$ spanned by $\left\{\widehat{x}^{k}: x \in E\right\}$, where $\widehat{x}^{k}(\gamma)=\gamma(x)^{k}$ (denoted $M_{k}$ in our previous proposition). Now define $g_{x}(\gamma)=1 /(1-\gamma(x))$ for each $x \in B_{E}^{\circ}$; the family $\left\{g_{x}\right\}_{x \in B_{E}^{\circ}}$ is linearly independent. Let $X$ be the closed subspace spanned by this family. $\mathcal{H}_{\mathrm{I}}\left(B_{E}^{\circ}\right)$ is the dual of $X$ : If $\alpha \in X^{\prime}$, extend $\alpha$ to all of $C\left(B_{E^{\prime}}, w^{*}\right)$ by Hahn-Banach preserving the norm (and the name). There 
is then a regular Borel measure $\mu$ on $\left(B_{E^{\prime}}, w^{*}\right)$ such that

$$
\alpha(h)=\int_{B_{E^{\prime}}} h(\gamma) d \mu(\gamma)
$$

for all $h \in C\left(B_{E^{\prime}}, w^{*}\right)$. Define

$$
f(x)=\alpha\left(g_{x}\right)=\int_{B_{E^{\prime}}} \frac{1}{1-\gamma(x)} d \mu(\gamma) .
$$

Then $f$ is integral, and $\|f\|_{\mathrm{I}} \leq\|\mu\|$ (for any $\mu$ representing $\alpha$ ). Thus we have $\|f\|_{\mathrm{I}} \leq\|\alpha\|$.

Now if $f \in \mathcal{H}_{\mathrm{I}}\left(B_{E}^{\circ}\right)$, define $\alpha$ on $s=\sum_{i=1}^{m} a_{i} g_{x_{i}}$ by

$$
\alpha(s)=\sum_{i=1}^{m} a_{i} f\left(x_{i}\right)
$$

Then for any $\mu$ representing $f$,

$$
|\alpha(s)|=\left|\sum_{i=1}^{m} a_{i} \int_{B_{E^{\prime}}} \frac{1}{1-\gamma\left(x_{i}\right)} d \mu(\gamma)\right|=\left|\int_{B_{E^{\prime}}} s(\gamma) d \mu(\gamma)\right| \leq\|\mu\|\|s\| .
$$

This shows that $\alpha$ is well defined and continuous. It also proves the integral representation. By continuity, $\alpha$ extends to $X$, with $\|\alpha\| \leq\|f\|_{\mathrm{I}}$. Thus $\mathcal{H}_{\mathrm{I}}\left(B_{E}^{\circ}\right)$ is the dual of $X$.

Now note that $X$ is the closed subspace of $C\left(B_{E^{\prime}}, w^{*}\right)$ spanned by $\left\{\widehat{x}^{k}\right.$ : $x \in E$ and $k=0,1, \ldots\}$, denoted $\bar{M}$ in the previous proposition. Indeed, clearly $X \subset \bar{M}$; but if $\mu$ is such that $\left.\mu\right|_{X}=0$, then

$$
0=\int_{B_{E^{\prime}}} \frac{1}{1-\gamma(x)} d \mu(\gamma)
$$

for all $x \in B_{E}^{\circ}$. By the Taylor expansion,

$$
0=\int_{B_{E^{\prime}}} \gamma(x)^{k} d \mu(\gamma)
$$

for all $x$ and $k$. Thus $\mu$ is zero over $M$, and therefore $X=\bar{M}$, so $\mathcal{H}_{\mathrm{I}}\left(B_{E}^{\circ}\right)$ is also the dual of $\bar{M}$. But [ACG] have shown that if $E$ has the approximation property, then $\bar{M}=A\left(B_{E^{\prime}}\right)$.

As with integral polynomials [CZ], if $E$ is a subspace of $G$, the predual $M(E)$ is a subspace of $M(G)$. Thus by Hahn-Banach integral functions are extendible, in the sense that if $f \in \mathcal{H}_{\mathrm{I}}\left(B_{E}^{\circ}\right)$, there is an $\bar{f} \in \mathcal{H}_{\mathrm{I}}\left(B_{G}^{\circ}\right)$, with $\left.\bar{f}\right|_{E}=f$ and $\|\bar{f}\|_{\mathrm{I}}=\|f\|_{\mathrm{I}}$. Moreover, as $\mathbb{C}$ is always isomorphic to a subspace of any complex Banach space $E$ we see that the disc algebra $A(\Delta)$ is isomorphic to a subspace of $M(E)$ and then $\mathcal{H}_{\mathrm{I}}\left(B_{\mathbb{C}}^{\circ}\right)$ is isomorphic to a quotient of $\mathcal{H}_{\mathrm{I}}\left(B_{E}^{\circ}\right)$. 
We now show that $\mathcal{H}_{\mathrm{I}}\left(B_{E}^{\circ}\right)$ is never separable. First we need the following proposition.

Proposition 6. Let $\phi: E \rightarrow F$ be a linear map with $\|\phi\| \leq 1$. The composition operator $C_{\phi}: \mathcal{H}_{\mathrm{I}}\left(B_{F}^{\circ}\right) \rightarrow \mathcal{H}_{\mathrm{I}}\left(B_{E}^{\circ}\right)$ given by $C_{\phi}(f)=f \circ \phi$ is a well defined linear and continuous mapping.

Proof. The transpose mapping of $\phi, \phi^{\prime}: F^{\prime} \rightarrow E^{\prime}$, also maps the unit ball $B_{F^{\prime}}$ into $B_{E^{\prime}}$ and is $w^{*}-w^{*}$ continuous. Therefore the composition operator $C_{\phi^{\prime}}: g \in C\left(B_{E^{\prime}}, w^{*}\right) \rightarrow g \circ \phi^{\prime} \in C\left(B_{F^{\prime}}, w^{*}\right)$ is a well defined linear and continuous mapping.

We then have $\left(C_{\phi^{\prime}}\right)^{\prime}: C\left(B_{F^{\prime}}, w^{*}\right)^{\prime} \rightarrow C\left(B_{E^{\prime}}, w^{*}\right)^{\prime}$ satisfying $\left(C_{\phi^{\prime}}\right)^{\prime}(\nu)(g)$ $=\nu\left(g \circ \phi^{\prime}\right)$ for all $g \in C\left(B_{E^{\prime}}, w^{*}\right)$ and all $\nu \in C\left(B_{F^{\prime}}, w^{*}\right)^{\prime}$. Given $f \in \mathcal{H}_{\mathrm{I}}\left(B_{F}^{\circ}\right)$ and $\nu$ a measure representing $f$ we have

$$
f(\phi(x))=\nu\left(g_{\phi(x)}\right)=\nu\left(g_{x} \circ \phi^{\prime}\right)=\left(C_{\phi^{\prime}}\right)^{\prime}(\nu)\left(g_{x}\right)
$$

for all $x \in B_{E}^{\circ}$. Therefore $f \circ \phi$ is an integral holomorphic function on $B_{E}^{\circ}$ and $\left(C_{\phi^{\prime}}\right)^{\prime}(\nu)$ is a measure representing it. Moreover,

$$
\|f \circ \phi\|_{\mathrm{I}} \leq\left\|\left(C_{\phi^{\prime}}\right)^{\prime}(\nu)\right\| \leq\left\|C_{\phi^{\prime}}\right\|\|\nu\| \leq\|\nu\|
$$

for all $\nu$ representing $f$. Thus $\|f \circ \phi\|_{\mathrm{I}} \leq\|f\|_{\mathrm{I}}$.

Corollary 7. (i) The Banach space $\mathcal{H}_{\mathrm{I}}\left(B_{E}^{\circ}\right)$ is never separable.

(ii) The integral polynomials are not a dense set in $\mathcal{H}_{\mathrm{I}}\left(B_{E}^{\circ}\right)$.

Proof. To begin with, pick $e \in E$ and $\Phi: E \rightarrow \mathbb{C}$ linear such that $\|e\|=1=\|\Phi\|=\Phi(e)$, and consider the norm one linear embedding $\phi$ : $z \in \mathbb{C} \rightarrow z e \in E$. The composition operator $C_{\phi}: \mathcal{H}_{\mathrm{I}}\left(B_{E}^{\circ}\right) \rightarrow \mathcal{H}_{\mathrm{I}}\left(B_{\mathbb{C}}^{\circ}\right)$ is also an onto mapping since for any $g \in \mathcal{H}_{\mathrm{I}}\left(B_{\mathbb{C}}^{\circ}\right)$, we have $g \circ \Phi \in \mathcal{H}_{\mathrm{I}}\left(B_{E}^{\circ}\right)$ and $C_{\phi}(g \circ \Phi)(z)=(g \circ \Phi \circ \phi)(z)=g(\Phi(z e))=g(z)$.

(i) Now, note that $\mathcal{H}_{\mathrm{I}}\left(B_{\mathbb{C}}^{\circ}\right)$ is non-separable as it is isomorphic to the dual of the disc algebra, which is known to be non-separable. Therefore, $\mathcal{H}_{\mathrm{I}}\left(B_{E}^{\circ}\right)$ cannot be separable.

(ii) If the integral polynomials were a dense subset of $\mathcal{H}_{\mathrm{I}}\left(B_{E}^{\circ}\right)$, its image under $C_{\phi}$ would also be a dense set in $\mathcal{H}_{\mathrm{I}}\left(B_{\mathbb{C}}^{\circ}\right)$. The proposition above ensures that for $P \in P_{\mathrm{I}}(E), P \circ \phi$ is an integral holomorphic function, hence an integral polynomial, thus $C_{\phi}\left(P_{\mathrm{I}}(E)\right) \subset P_{\mathrm{I}}(\mathbb{C})$. Moreover, as $P_{\mathrm{I}}(\mathbb{C}) \subset$ $\operatorname{span}\left\{1, z, z^{2}, \ldots, z^{n}, \ldots\right\}$ it would follow that this span is a dense set in $\mathcal{H}_{\mathrm{I}}\left(B_{\mathbb{C}}^{\circ}\right)$, against its non-separability.

REMARK 8. The above corollary shows that, in general, the Taylor series of a holomorphic integral function does not converge in $\mathcal{H}_{\mathrm{I}}\left(B_{E}^{\circ}\right)$ to the given function. This will prompt us to introduce, in the next section, another space of integral functions with better convergence properties. Even the Taylor series of an integral holomorphic function may not converge with respect to a coarser topology, like $w^{*}\left(\mathcal{H}_{\mathrm{I}}\left(B_{E}^{\circ}\right), A\left(B_{E^{\prime}}\right)\right)$. 
Let $E$ be a Banach space with the approximation property. Select $e \in E$ and $\varphi \in E^{*}$ with $\|\varphi\|=1=\|e\|=\varphi(e)$. We have $e \in E \subset A\left(B_{E^{\prime}}\right)$, which is a predual of $\mathcal{H}_{\mathrm{I}}\left(B_{E}^{\circ}\right)$. We consider the integral holomorphic function $f(x)=\sum_{k} \varphi(x)^{k}$ and we check that $\left(\varphi^{k}\right)$ is not weak ${ }^{*}$ null: Indeed, choose a sequence $\left(a_{m}\right)$ of scalars converging to 1 with $\left|a_{m}\right|<1$. Since $\lim _{m} a_{m} e=e$ in $A\left(B_{E^{*}}^{\circ}\right)$,

$$
\begin{aligned}
\left\langle\varphi^{k}, e\right\rangle & =\lim _{m}\left\langle\varphi^{k}, a_{m} e\right\rangle=\lim _{m} \int_{B_{E^{*}}^{\circ}} \gamma\left(a_{m} e\right)^{k} d \delta_{\varphi}(\gamma) \\
& =\lim _{m} \int_{B_{E^{*}}^{\circ}} a_{m}^{k} \gamma(e)^{k} d \delta_{\varphi}(\gamma)=\lim _{m} a_{m}^{k} \varphi(e)^{k}=1 .
\end{aligned}
$$

Thus $\left(\varphi^{k}\right)$ is not weak* null, so the Taylor series of $f$ is not $w^{*}\left(\mathcal{H}_{\mathrm{I}}\left(B_{E}^{\circ}\right)\right.$, $\left.A\left(B_{E^{\prime}}\right)\right)$ convergent.

REMARK 9. If $f=\sum_{k} P_{k}$ is a nuclear holomorphic function in the sense of $\sum_{k}\left\|P_{k}\right\|_{\mathrm{N}}<\infty$ ([D3, Definition 2.9]) then $f$ is also integral because $\left\|P_{k}\right\|_{\mathrm{I}} \leq\left\|P_{k}\right\|_{\mathrm{N}}([\mathrm{D} 3, \S 2.3])$. Moreover, not every integral function is nuclear even in the case when $P_{\mathrm{N}}(E)=P_{\mathrm{I}}(E)$, which holds for instance if $E^{*}$ has the Radon-Nikodym property ([D3, Proposition 2.27]), as the example in Remark 8 shows since $\left\|\varphi^{k}\right\|_{\mathrm{N}}=1$.

2. The spaces $\mathcal{H}_{\mathrm{bI}}\left(B_{E}^{\circ}\right)$ and $\mathcal{H}_{\mathrm{bI}}(E)$. In this section we define a Fréchet space of integral holomorphic functions over the open unit ball of $E$, in which the Taylor series expansion of an integral function converges to the function.

Definition 10. For any holomorphic function $f$ and any $0<r<1$ set $f_{r}(x)=f(r x)$. Then we define

$$
\mathcal{H}_{\mathrm{bI}}\left(B_{E}^{\circ}\right)=\left\{f \in \mathcal{H}\left(B_{E}^{\circ}\right): f_{r} \in \mathcal{H}_{\mathrm{I}}\left(B_{E}^{\circ}\right) \text { for all } 0<r<1\right\} .
$$

Also, define on this space the set of seminorms $p_{r}(f)=\left\|f_{r}\right\|_{\mathrm{I}}$.

Note that if $P$ is an integral $k$-homogeneous polynomial, $P_{r}(x)=P(r x)$ $=r^{k} P(x)$, and thus $p_{r}(P)=r^{k}\|P\|_{\mathrm{I}}$, so on the space $P_{\mathrm{I}}\left({ }^{k} E\right)$ all seminorms $p_{r}$ are equivalent to the integral norm. Hence the topology induced by $\mathcal{H}_{\mathrm{bI}}\left(B_{E}^{\circ}\right)$ on $P_{\mathrm{I}}\left({ }^{k} E\right)$ is the usual one. Note also that if $f \in \mathcal{H}_{\mathrm{bI}}\left(B_{E}^{\circ}\right)$, and $f=\sum_{k} P_{k}$ is its Taylor series expansion at 0 , then all $P_{k}$ 's are integral polynomials (indeed, for any $r, P_{k}=r^{-k} P_{k_{r}}$ ). We have the following characterization of $\mathcal{H}_{\mathrm{bI}}\left(B_{E}^{\circ}\right)$ in terms of the radius of integral convergence of $f=\sum_{k} P_{k}$,

$$
r_{\mathrm{I}}=\frac{1}{\lim \sup \left\|P_{k}\right\|_{\mathrm{I}}^{1 / k}} .
$$

Proposition 11. Let $f \in \mathcal{H}\left(B_{E}^{\circ}\right)$. Then $f \in \mathcal{H}_{\mathrm{bI}}\left(B_{E}^{\circ}\right)$ if and only if $P_{k} \in P_{\mathrm{I}}\left({ }^{k} E\right)$ and $r_{\mathrm{I}} \geq 1$. 
Proof. Take $f \in \mathcal{H}_{\mathrm{bI}}\left(B_{E}^{\circ}\right)$ and $r<1$. Then $f=\sum_{k} P_{k}$ implies $f_{r}=$ $\sum_{k} P_{k_{r}}$ and since this is an integral function, the sequence $\left(\left\|P_{k_{r}}\right\|_{\mathrm{I}}\right)$ is bounded, so

$$
1 \geq \lim \sup \left(\left\|P_{k_{r}}\right\|_{\mathrm{I}}\right)^{1 / k}=\lim \sup \left(r^{k}\left\|P_{k}\right\|_{\mathrm{I}}\right)^{1 / k}=r \lim \sup \left(\left\|P_{k}\right\|_{\mathrm{I}}\right)^{1 / k}=\frac{r}{r_{\mathrm{I}}} .
$$

Thus $r_{\mathrm{I}} \geq r$ for any $r<1$. So $r_{\mathrm{I}} \geq 1$.

Now if $r_{\mathrm{I}} \geq 1$, then $\sum_{k}\left\|P_{k}\right\|_{\mathrm{I}} r^{k}$ converges for all $r<1$. Thus $\left(r^{k}\left\|P_{k}\right\|_{\mathrm{I}}\right)_{k}$ $=\left(\left\|P_{k_{r}}\right\|_{\mathrm{I}}\right)_{k} \in \ell^{1}$, and $f_{r}=\sum_{k} P_{k_{r}}$ is integral for all $r<1$, by Proposition 3 . Hence, $f \in \mathcal{H}_{\mathrm{bI}}\left(B_{E}^{\circ}\right)$.

Note that since the usual polynomial norm is bounded above by the integral norm, we have $r_{\mathrm{I}} \leq r_{\mathrm{c}}$, the usual radius of convergence. Hence all functions of $\mathcal{H}_{\mathrm{bI}}\left(B_{E}^{\circ}\right)$ are of bounded type in the open unit ball of $E$. Note also that if $f \in \mathcal{H}_{\mathrm{I}}\left(B_{E}^{\circ}\right)$, then $f \in \mathcal{H}_{\mathrm{bI}}\left(B_{E}^{\circ}\right)$, for $f=\sum_{k} P_{k}$ with $\left(\left\|P_{k}\right\|_{\mathrm{I}}\right)$ bounded, so $\lim \sup \left(\left\|P_{k}\right\|_{\mathrm{I}}\right)^{1 / k} \leq 1$ and $r_{\mathrm{I}} \geq 1$. Something more can be said: if $f \in \mathcal{H}_{\mathrm{I}}\left(B_{E}^{\circ}\right)$ is represented by the measure $\mu$, then one may obtain measures representing $f_{r}$ as follows. If $A \subset r B_{E^{\prime}}$ is such that $A / r$ is measurable, define $\nu_{r}(A)=\mu(A / r)$. Then for all such $A$,

$$
\begin{aligned}
\int_{r B_{E^{\prime}}} \chi_{A}(\varphi) d \nu_{r}(\varphi) & =\nu_{r}(A)=\mu(A / r)=\int_{B_{E^{\prime}}} \chi_{A / r}(\gamma) d \mu(\gamma) \\
& =\int_{B_{E^{\prime}}} \chi_{A}(r \gamma) d \mu(\gamma)=\int_{B_{E^{\prime}}} \chi_{A}(\varphi) d \mu(\varphi / r) .
\end{aligned}
$$

Now if we set $\mu_{r}(U)=\nu_{r}\left(U \cap r B_{E^{\prime}}\right)=\mu\left(\frac{U}{r} \cap B_{E^{\prime}}\right)$ for any measurable $U \subset B_{E^{\prime}}$, we have

$$
\begin{aligned}
f_{r}(x) & =f(r x)=\int_{B_{E^{\prime}}} \frac{1}{1-\gamma(r x)} d \mu(\gamma)=\int_{B_{E^{\prime}}} \frac{1}{1-r \gamma(x)} d \mu(\gamma) \\
& =\int_{r B_{E^{\prime}}} \frac{1}{1-\varphi(x)} d \nu_{r}(\varphi)=\int_{B_{E^{\prime}}} \frac{1}{1-\gamma(x)} d \mu_{r}(\gamma),
\end{aligned}
$$

so that $\mu_{r}$ represents $f_{r}$. Note that $\left|\mu_{r}\right| \leq|\mu|$, so $\left\|f_{r}\right\|_{\mathrm{I}} \leq\|f\|_{\mathrm{I}}$ and the map $f \mapsto f_{r}$ is continuous in the integral norm, a fact which we shall use below. We now prove completeness of $\mathcal{H}_{\mathrm{bI}}\left(B_{E}^{\circ}\right)$.

Theorem 12. $\mathcal{H}_{\mathrm{bI}}\left(B_{E}^{\circ}\right)$ is a Fréchet space.

Proof. Clearly the topology of $\mathcal{H}_{\mathrm{bI}}\left(B_{E}^{\circ}\right)$ is given by a countable cofinal subset of the seminorms $p_{r}$. We check the completeness.

Let $\left(f_{n}\right)$ be a Cauchy sequence in $\mathcal{H}_{\mathrm{bI}}\left(B_{E}^{\circ}\right)$. Then for all $r<1,\left(f_{n_{r}}\right)$ is a Cauchy sequence in $\mathcal{H}_{\mathrm{I}}\left(B_{E}^{\circ}\right)$. Thus for each $r<1$ we have an integral holomorphic function $f_{(r)}$ with $f_{n_{r}} \rightarrow f_{(r)}$ in the integral norm. Now for any $s>r$, since $f_{n_{r}}=\left(f_{n_{s}}\right)_{r / s}$, continuity of the map $h \rightarrow h_{r}$ gives $f_{(r)}=f_{(s)_{r / s}}$. 
Then for any $x \in B_{E}^{\circ}$, and $r$ large enough, one has

$$
f_{(s)}\left(\frac{x}{s}\right)=f_{(s)}\left(\frac{r}{s} \frac{x}{r}\right)=f_{(s)_{r / s}}\left(\frac{x}{r}\right)=f_{(r)}\left(\frac{x}{r}\right) .
$$

There is then no ambiguity in setting $f(x)=f_{(r)}(x / r)$ for $r$ large enough; $f$ is easily seen to be holomorphic on $B_{E}^{\circ}$, and $f_{r}=f_{(r)}$ for all $r<1$, so $f \in \mathcal{H}_{\mathrm{bI}}\left(B_{E}^{\circ}\right)$. Also,

$$
p_{r}\left(f_{n}-f\right)=\left\|f_{n_{r}}-f_{r}\right\|_{\mathrm{I}}=\left\|f_{n_{r}}-f_{(r)}\right\|_{\mathrm{I}} \rightarrow 0
$$

as $n$ increases.

Lemma 13. If $f_{n} \rightarrow f$ in $\mathcal{H}_{\mathrm{bI}}\left(B_{E}^{\circ}\right)$, then $f_{n}$ converges uniformly to $f$ in $\varrho B_{E}$ for all $\varrho<1$.

Proof. For all $r<1$ we have $f_{n_{r}} \rightarrow f_{r}$ in $\mathcal{H}_{\mathrm{I}}\left(B_{E}^{\circ}\right)$. Now if $x \in B_{E}^{\circ}$ and $\|x\|<r<1$, then

$$
\begin{aligned}
\left|f_{n}(x)-f(x)\right| & =\left|f_{n_{r}}\left(\frac{x}{r}\right)-f_{r}\left(\frac{x}{r}\right)\right|=\left|\int_{B_{E^{\prime}}} \frac{1}{1-\gamma(x / r)} d\left(\mu_{n_{r}}-\mu_{r}\right)\right| \\
& \leq \frac{1}{1-\|x / r\|}\left\|f_{n_{r}}-f_{r}\right\|_{\mathrm{I}}=\frac{r}{r-\|x\|}\left\|f_{n_{r}}-f_{r}\right\|_{\mathrm{I}} \rightarrow 0
\end{aligned}
$$

as $n$ increases. Thus $f_{n}$ converges to $f$ uniformly on any ball of radius $\varrho<1$.

We now prove the convergence of the Taylor series in $\mathcal{H}_{\mathrm{bI}}\left(B_{E}^{\circ}\right)$.

Theorem 14. If $f \in \mathcal{H}_{\mathrm{bI}}\left(B_{E}^{\circ}\right)$, then its Taylor series at 0 converges to $f$ in $\mathcal{H}_{\mathrm{bI}}\left(B_{E}^{\circ}\right)$.

Proof. Let $\sum_{k} P_{k}$ be the Taylor series expansion of $f$ about 0 . Its partial sums form a Cauchy sequence; indeed, if $m>n$ and $r<s<1$, then

$$
\begin{aligned}
p_{r}\left(\sum_{k=0}^{m} P_{k}-\sum_{k=0}^{n-1} P_{k}\right) & =p_{r}\left(\sum_{k=n}^{m} P_{k}\right)=\left\|\sum_{k=n}^{m} P_{k_{r}}\right\|_{\mathrm{I}} \\
& \leq \sum_{k=n}^{m}\left\|P_{k_{r}}\right\|_{\mathrm{I}}=\sum_{k=n}^{m} r^{k}\left\|P_{k}\right\|_{\mathrm{I}}=\sum_{k=n}^{m}\left(\frac{r}{s}\right)^{k}\left\|P_{k_{s}}\right\|_{\mathrm{I}} \\
& \leq c \sum_{k=n}^{m}\left(\frac{r}{s}\right)^{k} \rightarrow 0
\end{aligned}
$$

as $n$ increases. Thus, by completeness of $\mathcal{H}_{\mathrm{bI}}\left(B_{E}^{\circ}\right)$, there is a function $h$ in $\mathcal{H}_{\mathrm{bI}}\left(B_{E}^{\circ}\right)$ such that $\sum_{k=0}^{\infty} P_{k}=h$. Since by Lemma 13 convergence in $\mathcal{H}_{\mathrm{bI}}\left(B_{E}^{\circ}\right)$ implies uniform convergence on $\varrho B_{E}$ for any $\varrho<1$, this $h$ can only be $f$. Thus $f$ 's Taylor series converges to $f$ in the topology of $\mathcal{H}_{\mathrm{bI}}\left(B_{E}^{\circ}\right)$. 
The concept of integral holomorphic function on the open unit ball of a Banach space $E$ can be generalized in the following way: given a convex balanced open subset $\Lambda$ of a Banach space $E$ we denote by $\mathcal{H}_{\mathrm{I}}(\Lambda)$ the Banach space of all holomorphic functions on $\Lambda$ such that there is a regular Borel measure $\mu$ on $\left(\Lambda^{\circ}, w^{*}\right)$ satisfying

$$
f(x)=\int_{\Lambda^{\circ}} \frac{1}{1-\gamma(x)} d \mu(\gamma)
$$

for every $x \in \Lambda$, endowed with the norm $\|f\|=\inf \{|\mu|: \mu$ represents $f\}$. As \|\|$\cdot\|\|$, the Minkowski gauge of $\Lambda$, is an equivalent norm on $E$ such that $B_{(E,\|\cdot \mid\|)}=\Lambda$, all properties obtained in Section 1 remain true for $\mathcal{H}_{\mathrm{I}}(\Lambda)$.

We will denote by $\mathcal{H}_{\mathrm{bI}}(E)$ the Fréchet space of all entire functions $f$ on the Banach space $E$ whose restrictions to $n B_{E}$ belong to $\mathcal{H}_{\mathrm{I}}\left(n B_{E}\right)$ for $n=1,2, \ldots$, endowed with the sequence $\left(p_{n}\right)_{n}$ of norms defined as

$$
p_{n}(f)=\left\|\left.f\right|_{n B_{E}}\right\|_{\mathrm{I}}, \quad n=1,2, \ldots
$$

3. Biduality and reflexivity. According to [GMR] a sequence of Banach spaces $\left(E_{k},\|\cdot\|\right)_{k}$ is an $R$-Schauder decomposition $(0<R \leq \infty)$ of a Fréchet space $E$ if it is a Schauder decomposition of $E$ and for every sequence $\left(x_{k}\right)_{k}, x_{k} \in E_{k}$, the series $\sum_{k=0}^{\infty} x_{k}$ converges in $E$ if and only if $\limsup _{k}\left\|x_{k}\right\|_{k}^{1 / k} \leq 1 / R$. By Proposition 11, $\left(P_{\mathrm{I}}\left({ }^{k} E\right)\right)_{k}$ is a 1-Schauder decomposition of $\mathcal{H}_{\mathrm{bI}}\left(B_{E}^{\circ}\right)$. Analogously it can be proved that $\left(P_{\mathrm{I}}\left({ }^{k} E\right)\right)_{k}$ is an $\infty$-Schauder decomposition of $\mathcal{H}_{\mathrm{bI}}(E)$. Hence, by [GMR, Remark 5], $\mathcal{H}_{\mathrm{bI}}\left(B_{E}^{\circ}\right)$ and $\mathcal{H}_{\mathrm{bI}}(E)$ are not topologically isomorphic spaces when $E \neq\{0\}$.

The space $\mathcal{H}_{\mathrm{I}}\left(B_{E}^{\circ}\right)$ is never reflexive. The disc algebra $A(\Delta)$ is not a reflexive space, and so by Theorem $5, \mathcal{H}_{\mathrm{I}}\left(B_{\mathbb{C}}^{\circ}\right)$ is not a reflexive Banach space. But for any Banach space $E, \mathcal{H}_{\mathrm{I}}\left(B_{\mathbb{C}}^{\circ}\right)$ is isomorphic to a quotient of $\mathcal{H}_{\mathrm{I}}\left(B_{E}^{\circ}\right)$, hence the space $\mathcal{H}_{\mathrm{I}}\left(B_{E}^{\circ}\right)$ is never reflexive. The situation is quite different for $\mathcal{H}_{\mathrm{bI}}\left(B_{E}^{\circ}\right)$ and $\mathcal{H}_{\mathrm{bI}}(E)$ as we will show in the proposition below.

In [GMR, Theorem 8], it is proved that if $\left(E_{n},\|\cdot\|_{n}\right)_{n}$ is an $R$-Schauder decomposition of $E, 0<R \leq \infty$, then $\left(E_{n}^{\prime \prime},\|\cdot\|_{n}^{\prime \prime}\right)_{n}$ is (canonically) an $R$-Schauder decomposition of $E^{\prime \prime}$. Hence we have the following result.

Proposition 15. Let $E$ be a Banach space. The spaces $\mathcal{H}_{\mathrm{bI}}\left(B_{E}\right)$ and $\mathcal{H}_{\mathrm{bI}}(E)$ are reflexive if and only if $P_{\mathrm{I}}\left({ }^{k} E\right)$ is a reflexive space for every $k=1,2, \ldots$

As $E^{\prime}=P_{\mathrm{I}}\left({ }^{1} E\right)$ we have to assume that $E$ is a reflexive Banach space in order to find examples satisfying the above proposition.

Note that if $E$ is a reflexive Banach space with the approximation property, then the dual space of $P_{\mathrm{I}}\left({ }^{k} E\right)$ is isometric to $P\left({ }^{k} E^{\prime}\right)$. Indeed, since $E^{\prime}$ 
has the Radon-Nikodym property, the space of integral polynomials is isometric to that of nuclear polynomials ([CD], $[\mathrm{BR}])$, and since $E^{\prime}$ has the approximation property, $P_{\mathrm{N}}\left({ }^{k} E\right)^{\prime}=P\left({ }^{k} E^{\prime}\right)([\mathrm{G}])$. Thus, in this situation, $P_{\mathrm{I}}\left({ }^{k} E\right)$ is reflexive if and only if $P\left({ }^{k} E^{\prime}\right)$ is reflexive.

If $T^{*}$ is the original Tsirelson space, $P\left({ }^{k} T^{*}\right)$ is reflexive for all $k$ ([AAD]). Hence $\left(P_{\mathrm{I}}\left({ }^{k} T\right)\right)_{k}$ is a sequence of reflexive Banach spaces, thus $\mathcal{H}_{\mathrm{bI}}\left(B_{T}\right)$ and $\mathcal{H}_{\mathrm{bI}}(T)$ are reflexive. Note that for any $k \geq 2, P\left({ }^{k} T\right)$ is not a reflexive Banach space and hence neither $\mathcal{H}_{\mathrm{bI}}\left(B_{T^{*}}\right)$ nor $\mathcal{H}_{\mathrm{bI}}\left(T^{*}\right)$ are reflexive spaces.

Now we turn to the study of the bidual of the spaces of integral holomorphic functions. In recent years biduality and reflexivity of spaces of polynomials have been extensively studied by a number of authors ([AD], [D4], [JPZ], [JM], [Ve], [V1]). Our next two propositions and corollary are the dual versions of [D3, Proposition 2.45, Corollary 2.46 and Propositions 2.47 and 2.48]. We refer to [D3] for the definition and properties of Q-reflexive Banach spaces.

Proposition 16. Let $E$ be a Q-reflexive Banach space such that $E^{\prime \prime \prime}$ has the approximation property and $P_{w}\left({ }^{k} E^{\prime \prime}\right)=P\left({ }^{k} E^{\prime \prime}\right)$ for all $k=1,2, \ldots$ Then $P_{\mathrm{I}}\left({ }^{k} E^{\prime}\right)$ is isomorphic to $P_{\mathrm{I}}\left({ }^{k} E^{\prime \prime \prime}\right)$ for all $k=1,2 \ldots$

Proof. Since $E^{\prime}$ has the approximation property the dual of $P_{w}\left({ }^{k} E\right)$ is isometric to $P_{\mathrm{I}}\left({ }^{k} E^{\prime}\right)$. As $E$ is Q-reflexive, by [D3, Definition 2.44 and Proposition 2.45] we see that $P_{w}\left({ }^{k} E\right)=P\left({ }^{k} E\right)$ as sets and that $P_{w}\left({ }^{k} E^{\prime \prime}\right)$ is canonically isomorphic to $P\left({ }^{k} E\right)^{\prime \prime}$. By hypothesis $P_{w}\left({ }^{k} E^{\prime \prime}\right)=P\left({ }^{k} E^{\prime \prime}\right)$ and $E^{\prime \prime \prime}$ has the approximation property, thus $P\left({ }^{k} E^{\prime \prime}\right)^{\prime}=P_{\mathrm{I}}\left({ }^{k} E^{\prime \prime \prime}\right)$ isometrically, hence we have the chain

$$
P_{\mathrm{I}}\left({ }^{k} E^{\prime}\right)^{\prime \prime} \cong P\left({ }^{k} E^{\prime \prime}\right)^{\prime}=P_{w}\left({ }^{k} E^{\prime \prime}\right)^{\prime}=P_{\mathrm{I}}\left({ }^{k} E^{\prime \prime \prime}\right) .
$$

We need to improve the above result in a way that allows us to control the norms of the isomorphisms between $P_{\mathrm{I}}\left({ }^{k} E^{\prime}\right)^{\prime \prime}$ and $P_{\mathrm{I}}\left({ }^{k} E^{\prime \prime \prime}\right)$ in order to obtain results for spaces of holomorphic functions of integral type. We do this in the next proposition.

Proposition 17. Let $E$ be a Q-reflexive Banach space such that $E^{\prime \prime \prime}$ has the approximation property, $P_{w}\left({ }^{k} E^{\prime \prime}\right)=P\left({ }^{k} E^{\prime \prime}\right)$ for all $k=1,2, \ldots$ and the space $\ell_{1}$ is not contained in $\widehat{\bigotimes}_{k, s, \varepsilon} E^{\prime}$ (in particular if $E^{\prime \prime}$ has the Radon-Nikodym property). Then the space $P_{\mathrm{I}}\left({ }^{k} E^{\prime}\right)$ is isometric to $P_{\mathrm{I}}\left({ }^{k} E^{\prime \prime \prime}\right)$ for all $k=1,2 \ldots$

Proof. By $[\mathrm{CD}],[\mathrm{BR}]$ the space $P_{\mathrm{I}}\left({ }^{k} E^{\prime}\right)$ coincides with the space of nuclear polynomials $P_{\mathrm{N}}\left({ }^{k} E^{\prime}\right)$ and moreover the identity is an isometric isomorphism. As $E^{\prime \prime}$ has the approximation property we have $\widehat{\bigotimes}_{k, s, \pi} E^{\prime}=P_{\mathrm{N}}\left({ }^{k} E^{\prime}\right)$ isometrically and then $P_{\mathrm{N}}\left({ }^{k} E^{\prime}\right)^{\prime}=P\left({ }^{k} E^{\prime \prime}\right)$ isometrically. Thus we have the 
following chain of isometries:

$$
P_{\mathrm{I}}\left({ }^{k} E^{\prime}\right)^{\prime \prime}=P_{\mathrm{N}}\left({ }^{k} E^{\prime}\right)^{\prime \prime}=P\left({ }^{k} E^{\prime \prime}\right)^{\prime}=P_{w}\left({ }^{k} E^{\prime \prime}\right)^{\prime}=P_{\mathrm{I}}\left({ }^{k} E^{\prime \prime \prime}\right) .
$$

COROLlary 18. Let $E$ be a Q-reflexive Banach space such that $E^{\prime \prime}$ has the Radon-Nikodym property, $E^{\prime \prime \prime}$ has the approximation property and $P_{w}\left({ }^{k} E^{\prime \prime}\right)=P\left({ }^{k} E^{\prime \prime}\right)$ for all $k=1,2, \ldots$ Then the space $\mathcal{H}_{\mathrm{bI}}\left(E^{\prime}\right)^{\prime \prime}$ is isomorphic to $\mathcal{H}_{\mathrm{bI}}\left(E^{\prime \prime \prime}\right)$ and the space $\mathcal{H}_{\mathrm{bI}}\left(B_{E^{\prime}}^{\circ}\right)^{\prime \prime}$ is isomorphic to $\mathcal{H}_{\mathrm{bI}}\left(B_{E^{\prime \prime \prime}}^{\circ}\right)$.

Proof. $P_{\mathrm{I}}\left({ }^{k} E^{\prime}\right)^{\prime \prime}$ is isometric to $P_{\mathrm{I}}\left({ }^{k} E^{\prime \prime \prime}\right)$ for all $k=1,2, \ldots$ Then the conclusion follows from [GMR, Theorem 9].

To give an example of a Banach space satisfying the hypothesis of the above corollary we need the following lemma.

Lemma 19. Let $E$ a quasi-reflexive Banach space such that $P_{w}\left({ }^{k} E\right)=$ $P\left({ }^{k} E\right)$. Then $P_{w}\left({ }^{k} E^{\prime \prime}\right)=P\left({ }^{k} E^{\prime \prime}\right)$.

Proof. By hypothesis $E$ has finite codimension in its bidual $E^{\prime \prime}$. Consider a finite-dimensional subspace $F$ of $E^{\prime \prime}$ such that $E^{\prime \prime}=E \oplus F$; this is a topological decomposition for the topology of the norm. We denote by $p$ the projection, and by $r$ the maximum of the norms of $p$ and Id $-p$. Let $P \in P\left({ }^{k} E^{\prime \prime}\right)$. As $P$ is uniformly continuous on $r B_{E^{\prime \prime}}$, given $\varepsilon>0$ there exists a $\delta>0$ such that $\|P(z)-P(u)\|<\varepsilon$ for all $z, u \in r B_{E^{\prime \prime}}$ with $\|z-u\|<\delta$. Consider a net $\left\{z_{\alpha}, \alpha \in \mathcal{A}, \leq\right\}$ in $B_{E^{\prime \prime}}$, the closed unit ball of $E^{\prime \prime}$, weakly convergent to $z_{0} \in B_{E^{\prime \prime}}$. Let $x_{\alpha}=p\left(z_{\alpha}\right)$ and $y_{\alpha}=z_{\alpha}-p\left(z_{\alpha}\right)$ for all $\alpha \in \mathcal{A}, x_{0}=p\left(z_{0}\right)$ and $y_{0}=z_{0}-p\left(z_{0}\right)$. We have $\left\|x_{0}\right\| \leq r,\left\|y_{0}\right\| \leq r$, $\left\|x_{\alpha}\right\| \leq r,\left\|y_{\alpha}\right\| \leq r$ for all $\alpha$. As $E^{\prime \prime}=E \oplus F$, topologically also for the $w\left(E^{\prime \prime}, E^{\prime \prime \prime}\right)$ topology, $\left\{x_{\alpha}, \alpha \in \mathcal{A}, \leq\right\}$ weakly converges to $x_{0}$ in $E$ and $\left\{y_{\alpha}, \alpha \in \mathcal{A}, \leq\right\}$ weakly converges to $y_{0}$ in $F$. But $F$ is finite-dimensional, thus $\left\{y_{\alpha}, \alpha \in \alpha, \leq\right\}$ norm converges to $y_{0}$ in $F$. Hence we can find $\alpha_{0} \in \mathcal{A}$ such that $\left\|y_{\alpha}-y_{0}\right\|<\delta$ for all $\alpha \geq \alpha_{0}$ and then $\left|P\left(z_{\alpha}\right)-P\left(x_{\alpha}+y_{0}\right)\right|<\varepsilon$ for all $\alpha \geq \alpha_{0}$.

Let $\check{P}$ be the symmetric multilinear form associated to $P$. If we define $Q_{n}: E \rightarrow \mathbb{C}$ by $Q_{n}(x):=\check{P}\left(x^{n}, y_{0}^{k-n}\right)$ for all $x \in E$ and every $n=1, \ldots, k$, we have $Q_{n} \in P\left({ }^{n} E\right)=P_{w}\left({ }^{n} E\right)$ for $n=1, \ldots, k$. Then $\left\{Q_{n}\left(x_{\alpha}\right), \alpha \in \mathcal{A}, \leq\right\}$ converges to $Q_{n}\left(x_{0}\right)$ for $n=1, \ldots, k$ and we can find $\alpha_{n}$ with $\mid Q_{n}\left(x_{\alpha}\right)-$ $Q_{n}\left(x_{0}\right) \mid<\varepsilon$ for $\alpha \geq \alpha_{n}, n=1, \ldots, k$. Consider $\beta \geq \alpha_{n}$ for $n=0,1, \ldots, k$. We have

$$
\begin{aligned}
\left|P\left(z_{\alpha}\right)-P\left(z_{0}\right)\right| & \leq\left|P\left(z_{\alpha}\right)-P\left(x_{\alpha}+y_{0}\right)\right|+\left|P\left(x_{\alpha}+y_{0}\right)-P\left(z_{0}\right)\right| \\
& \leq \varepsilon+\sum_{n=1}^{k}\left|\check{P}\left(x_{\alpha}^{n}, y_{0}^{k-n}\right)-\check{P}\left(x_{0}^{n}, y_{0}^{k-n}\right)\right|<2^{k} \varepsilon
\end{aligned}
$$

whenever $\alpha \geq \beta$. Hence $P \in P_{w}\left({ }^{k} E^{\prime \prime}\right)$. 
The best known example of a non-reflexive Q-reflexive Banach space is the Tsirelson-James space $T_{J}^{*}\left([\mathrm{AD}]\right.$, [D3, Example 2.43]). As $T_{J}^{*}$ and all its higher duals are quasi-reflexive and moreover have a basis, the higher duals have the approximation property and the Radon-Nikodym property. Now by applying Lemma 19 and Corollary 18 we obtain the following.

Corollary 20. $\mathcal{H}_{\mathrm{bI}}\left(T_{J}^{* \prime}\right)^{\prime \prime} \cong \mathcal{H}_{\mathrm{bI}}\left(T_{J}^{* \prime \prime \prime}\right)$ and $\mathcal{H}_{\mathrm{bI}}\left(B_{T_{J}^{*}}^{\circ}{ }^{\prime \prime}\right) \cong \mathcal{H}_{\mathrm{bI}}\left(B_{T_{J}^{* \prime \prime}}^{\circ}\right)$.

Remark 21. A sequence $\left(x_{n}\right)$ in a Banach space $E$ is called $\tau_{\alpha}$-convergent to zero (for $0<\alpha<1$ ) if there is a $c \geq 0$ such that $\left\|\sum_{n \in B} x_{n}\right\| \leq c|B|^{\alpha}$ for all finite $B \subset \mathbb{N}$, where $|B|$ is the number of elements of $B$. A Banach space $E$ is said to have property $P_{\alpha}$ if every weak-null sequence admits a $\tau_{\alpha^{-}}$ convergent subsequence. The concept of $\tau_{\alpha}$-convergence is due to Pełczyński $[\mathrm{P}]$. If $E$ is a quasi-reflexive Banach space which has property $P_{\alpha}$, then $E^{\prime \prime}$ also has property $P_{\alpha}$. Indeed, let $\left(x_{n}^{\prime \prime}\right) \subset E^{\prime \prime}$ be a $w\left(E^{\prime \prime}, E^{\prime \prime \prime}\right)$-null sequence. Consider a finite-dimensional subspace $F$ of $E^{\prime \prime}$ such that $E^{\prime \prime}=E \oplus F$; this is a topological decomposition for the weak topology. Hence there exist weaknull sequences $\left(x_{n}\right) \subset E$ and $\left(y_{n}\right) \subset F$ such that $x_{n}^{\prime \prime}=x_{n}+y_{n}$ for all $n \in \mathbb{N}$. As $\left(y_{n}\right)$ also converges in norm, we can assume, by taking a subsequence if necessary, that $\left\|y_{n}\right\| \leq 1 / 2^{n}$ for all $n \in \mathbb{N}$. Since $E$ has property $P_{\alpha}$ there exists a subsequence $\left(x_{n_{k}}\right)$ and $c \geq 0$ such that $\left\|\sum_{k \in B} x_{n_{k}}\right\| \leq c|B|^{\alpha}$ for all finite $B \subset \mathbb{N}$. Hence

$$
\left\|\sum_{k \in B} x_{n_{k}}^{\prime \prime}\right\| \leq\left\|\sum_{k \in B} x_{n_{k}}\right\|+\left\|\sum_{k \in B} y_{n_{k}}\right\| \leq c|B|^{\alpha}+1 \leq(c+1)|B|^{\alpha}
$$

for all finite $B \subset \mathbb{N}$.

There are examples of Q-reflexive Banach spaces which are not quasireflexive. In Theorem 5 of [V2] it is proved that if $Y$ is a closed subspace of $T^{*}$ with the approximation property, then $X:=Y \widehat{\otimes}_{\pi} T_{J}^{*}$ is Q-reflexive and moreover that $X^{\prime}=Y \widehat{\otimes}_{\varepsilon} T_{J}^{* \prime}$ and $X^{\prime \prime}=Y \widehat{\otimes}_{\pi} T_{J}^{* \prime \prime}$. We have the following.

THEOREM 22. Let $Y$ be a closed subspace of $T^{*}$ with the approximation property. If $X:=Y \widetilde{\otimes}_{\pi} T_{J}^{*}$, then $\mathcal{H}_{\mathrm{bI}}\left(X^{\prime}\right)^{\prime \prime} \cong \mathcal{H}_{\mathrm{bI}}\left(X^{\prime \prime \prime}\right)$ and $\mathcal{H}_{\mathrm{bI}}\left(B_{X^{\prime}}^{\circ}\right)^{\prime \prime} \cong$ $\mathcal{H}_{\mathrm{bI}}\left(B_{X^{\prime \prime \prime}}^{\circ}\right)$.

Proof. The proof of Theorem 5 in [V2] shows that $X^{\prime}=\mathcal{L}_{w}\left(Y, T_{J}^{*}\right)=$ $\mathcal{L}_{w^{*}}\left(Y^{\prime \prime}, T_{J}^{* \prime \prime}\right)$, which by Theorem 1 in [V1] is an Asplund space, that is, $X^{\prime \prime}$ has the Radon-Nikodym Property. Now we follow the arguments in that proof to check the remaining assumptions in Corollary 18 for $X$.

By [AF1, Corollary 3.2], if $F$ and $G$ are two Banach spaces that have property $P_{\alpha}$ for all $\alpha>0$, then all $2 n$-linear continuous mappings $F^{n} \times G^{n} \rightarrow$ $\mathbb{C}$ are weakly sequentially continuous. If moreover $\ell_{1} \not \subset F^{n} \times G^{n}$, they are weakly continuous on bounded subsets of $F^{n} \times G^{n}$. Hence, by [V2, Lemma 2], 
$P_{w}\left({ }^{k} F \widehat{\otimes}_{\pi} G\right)=P\left({ }^{k} F \widehat{\otimes}_{\pi} G\right)$. All closed subspaces of Tsirelson's space $T^{*}$ and the space $T_{J}^{*}$ have property $P_{\alpha}$ for all $\alpha>0$ ([AF2]). Now, by Remark $21, G=T_{J}^{* \prime \prime}$ has property $P_{\alpha}$ as well for all $\alpha>0$. As $X^{\prime \prime}=Y \widehat{\otimes}_{\pi} T_{J}^{* \prime \prime}$, the above comment leads to $P_{w}\left({ }^{k} X^{\prime \prime}\right)=P\left({ }^{k} X^{\prime \prime}\right)$ for all $k=1,2, \ldots$ and also to proving that all bilinear mappings on $Y \times T_{J}^{* \prime \prime}$ are weakly continuous on bounded sets; thus $X^{\prime \prime \prime}=\left(Y \widehat{\otimes}_{\pi} T_{J}^{* \prime \prime}\right)^{\prime}=Y^{\prime} \widehat{\otimes}_{\varepsilon} T_{J}^{* \prime \prime \prime}$ since both $Y^{\prime}$ and $T_{J}^{* \prime \prime \prime}$ have the approximation property, which in turn implies that $X^{\prime \prime \prime}$ has the approximation property.

Final comments. We end with some informal comments and open problems. In the one-dimensional case, we had

$$
f(x)=\int_{T} \frac{1}{1-\omega x} f(1 / \omega) \frac{d \omega}{2 \pi i \omega} \text { and }\langle f, g\rangle=\int_{\Gamma^{-1}} g(\omega) f(1 / \omega) \frac{d \omega}{2 \pi i \omega} .
$$

Two questions immediately come to mind regarding the integral representation of holomorphic functions on Banach spaces.

(i) Should we be integrating over $B_{E^{\prime}}$ or over some smaller subset $K$ ?

(ii) Can we describe $\mu$ in terms of $f$ and some "universal" measure $P$ ? (That is, $d \mu(\gamma)=\widehat{f}(\gamma) d P(\gamma)$; note that $d \omega /(2 \pi i \omega)$ is a probability measure on $T$.)

We want elements of the dual of $A\left(B_{E^{\prime}}\right)$ to provide us with measures. Thus we need $A\left(B_{E^{\prime}}\right)$ to be a subspace of $C(K)$, with $K \subset B_{E^{\prime}}$, so functions in $A\left(B_{E^{\prime}}\right)$ should attain their norm on $K$, that is, we want $K$ to be a $w^{*}$ closed boundary for $A\left(B_{E^{\prime}}\right)$. The unit sphere of $E^{\prime}$ is not $w^{*}$-closed, and hence not a good candidate. The Shilov boundary of $A\left(B_{E^{\prime}}\right)$ is a better candidate.

One interesting case is $E=\ell^{1}\left(E^{\prime}=\ell^{\infty}\right.$ and $\left.B_{E^{\prime}}=\Delta^{\mathbb{N}}\right)$. Here the Shilov boundary of $A\left(B_{E^{\prime}}\right)$ is $T^{\mathbb{N}}$, on which one has the rotation-invariant probability measure $P=$ product of normalized Lebesgue measure on $T$. We can then write, for $x \in B_{\ell^{1}}$,

$$
f(x)=\int_{T^{\mathbb{N}}} \frac{1}{1-\gamma(x)} \widehat{f}(\gamma) d P(\gamma) .
$$

Of course any integrable $\widehat{f}$ will give us an integral holomorphic function $f$. Given $f$, we can find the corresponding $\widehat{f}$ in only a few elementary cases, such as

$$
x_{1} x_{2}^{2}=\int_{T^{\mathbb{N}}} \frac{1}{1-\gamma(x)} \bar{\gamma}_{1} \bar{\gamma}_{2}^{2} d P(\gamma)
$$

(see also $[R]$ ). 


\section{References}

[A] R. Alencar, Multilinear mappings of nuclear and integral type, Proc. Amer. Math. Soc. 94 (1985), 33-38.

[AAD] R. Alencar, R. M. Aron and S. Dineen, A reflexive space of holomorphic functions in infinitely many variables, ibid. 90 (1984), 407-411.

[AF1] R. Alencar and K. Floret, Weak-strong continuity of multilinear mappings and the Petczyński-Pitt theorem, J. Math. Anal. Appl. 206 (1997), 532-546.

[AF2] - - , Weak continuity of multilinear mappings on Tsirelson's space, Quaest. Math. 21 (1998), 177-186.

[ACG] R. M. Aron, B. J. Cole and T. W. Gamelin, Weak-star continuous analytic functions, Canad. J. Math. 47 (1995), 673-683.

[AD] R. M. Aron and S. Dineen, Q-reflexive Banach spaces, Rocky Mountain J. Math. 27 (1997), 1009-1025.

[BR] C. Boyd and R. Ryan, Geometric theory of spaces of integral polynomials and symmetric tensor products, J. Funct. Anal. 179 (2001), 18-42.

[CD] D. Carando and V. Dimant, Duality in spaces of nuclear and integral polynomials, J. Math. Anal. Appl. 241 (2000), 107-121.

[CZ] D. Carando and I. Zalduendo, A Hahn-Banach theorem for integral polynomials, Proc. Amer. Math. Soc. 127 (1999), 241-250.

[D1] S. Dineen, Holomorphic functions on a Banach space, Bull. Amer. Math. Soc. 76 (1970), 883-886.

[D2] -, Holomorphy types on a Banach space, Studia Math. 39 (1971), 241-288.

[D3] - Complex Analysis on Infinite-Dimensional Spaces, Springer, 1999.

[D4] - Canonical mappings for polynomials and holomorphic functions on Banach spaces, Proc. Amer. Math. Soc. 129 (2001), 2897-2905.

[GMR] P. Galindo, M. Maestre and P. Rueda, Biduality in spaces of holomorphic functions, Math. Scand. 86 (2000), 5-16.

[G] C. P. Gupta, On the Malgrange theorem for nuclearly entire functions of bounded type on a Banach space, Notas Mat. 37, Inst. Mat. Pura Apl., Río de Janeiro, 1968.

[JM] J. A. Jaramillo and L. A. Moraes, Duality and reflexivity in spaces of polynomials, Arch. Math. (Basel) 74 (2000), 282-293.

[JPZ] J. A. Jaramillo, A. Prieto and I. Zalduendo, The bidual of a space of polynomials on a Banach space, Math. Proc. Cambridge Philos. Soc. 122 (1997), 457-471.

[K] G. Köthe, Dualität in der Funktionentheorie, J. Reine Angew. Math. 191 (1953), 30-49.

[P] A. Pełczyński, A property of multilinear operations, Studia Math. 16 (1957), 173-182.

[R] R. Ryan, Holomorphic mappings on $\ell_{1}$, Trans. Amer. Math. Soc. 302 (1987), 797-811.

[S] J. Sebastião e Silva, As funções analíticas e a análise functional, Portugal. Math. 9 (1950), 1-130.

[V1] M. Valdivia, Banach spaces of polynomials without copies of $\ell_{1}$, Proc. Amer. Math. Soc. 123 (1995), 3143-3150.

[V2] - Some properties in spaces of multilinear functionals and spaces of polynomials, Math. Proc. Roy. Irish Acad. 98A (1998), 87-106. 
[Ve] M. Venkova, Properties of Q-reflexive Banach spaces, J. Math. Anal. Appl. 264 (2001), 96-106.
V. Dimant
Departamento de Matemática
Universidad de San Andrés
CC35-1644 Victoria
Buenos Aires, Argentina
E-mail: vero@udesa.edu.ar
I. Zalduendo
Departamento de Matemática
Universidad Torcuato Di Tella
Miñones 2177 (C1428ATG)
Buenos Aires, Argentina
E-mail: izalduendo@utdt.edu

\author{
P. Galindo, M. Maestre \\ Departamento de Análisis Matemático \\ Universidad de Valencia \\ 46100 Burjasot \\ Valencia, Spain \\ E-mail: pablo.galindo@uv.es \\ manuel.maestre@uv.es
}

Received October 8, 2002

Revised version April 4, 2003 